\title{
Statistical Guidelines for Publishing in the Journal of Youth and Adolescence
}

\author{
Roger J. R. Levesque ${ }^{1}$
}

Received: 19 October 2015 / Accepted: 19 October 2015/Published online: 28 October 2015

(C) Springer Science+Business Media New York 2015

Advances in developmental science and the Journal of Youth and Adolescence's rapid growth urge us to provide statistical guidelines for prospective authors. In terms of the Journal, more guidance is needed because we have moved away from supporting purely qualitative work, have experienced an increase in submissions, continue to reach out to authors who do not typically publish in developmental journals, and receive many inquiries from authors wondering whether their studies fall within the Journal's mission. In terms of statistical and methodological advances in our field, more guidance appears needed given concerns about statistical power, interest in replication, and the overall desire to develop a more robust science. These changes spur efforts to be more proactive in helping authors develop stronger manuscripts and make more informed decisions about submitting them.

Before proceeding, however, it is important to emphasize that guidelines are just that: guidelines. Publication decisions, which rest on the projected impact of manuscripts, depend on several factors, including how to consider the guidelines. Yet, overall, we expect that considering the guidelines will both increase the likelihood of getting published in the Journal and of contributing to better developmental science, particularly when authors join us from disciplines with other expectations.

Roger J. R. Levesque

rlevesqu@indiana.edu

Indiana University, Bloomington, IN, USA

\section{Describe Data Efficiently}

Rich descriptions help readers understand findings. Especially helpful is reporting basic statistics regarding the sample size, particularly if the size varies across analyses. Also helpful are basic analyses, like correlation tables of key variables, that allow readers to identify trends and make their own evaluations. Such analyses should be done in a manner that moves the manuscript's narrative forward; e.g., they present correlations by sex, age, race or any other grouping when the study centers on those groups' differences/similarities. Also important to report are appropriate measures of variability around means and around effects; e.g., this can involve reporting confidence intervals around means and/or around standardized effect sizes. While it is not advisable to overwhelm readers with data, it is advisable to provide them with enough information so that they can evaluate the study and, eventually, replicate it.

\section{Address Statistical Power}

Effective methods sections state what criteria were used to determine the sample size needed for the conducted analyses. These criteria address issues of statistical power, which refers to the probability that a test will reject a false null hypothesis. Studies with low statistical power inherently produce ambiguous results that often fail to replicate. This potential failure means that analyses should be conducted with ample statistical power, with authors reporting their estimate of a priori power, not post hoc power. While some analyses need not provide such reports, given the nature of their analyses and sample sizes, readers benefit from authors' addressing potential concerns about statistical power. Determinations of adequate power call for 
flexibility, and even low power can be offset by other considerations, such as novelty and the difficulty of obtaining larger (and still relevant) samples. Again, persuasive narratives that draw on strong theory and appropriate hypotheses can help sway reviewers and future readers. Science is about the provisional provision of answers; the goal is to make reasonable contributions to the development of answers and questions.

\section{Avoid Multiple Statistical Tests}

Multiple statistical tests are inherently suspect and problematic. Repeatedly used tests of statistical significance, such as analysis of variance and t-tests, increase Type I error rates (i.e., they lead to false positives-they falsely reject the [true] null hypothesis). As a result, they should be run with statistical corrections, such as Bonferroni corrections. The same need to adjust arises with more advanced statistical analyses. For example, authors now increasingly include multiple covariates in their analyses, and such practices are encouraged to ensure proper model specifications. But, the use of covariates should be planned in advance or described as exploratory. When exploratory, analyses must acknowledge possible inflation of the Type I error rates. Best practice also includes providing foundations for the use of covariates. Best practice avoids analyzing data without covariates and then running analyses again with covariates and reporting only the latter as confirming hypotheses.

The above examples of suspect practices can be obvious; several others can be more subtle. For example, running multiple analyses on multiple dependent variables provides another example of problematic practices. Such analyses increase the possibility that chance alone will lead some of the analyses to produce significant results. Reporting the dependent variables that were analyzed is important for readers to make sense of the findings. It also is important to justify using subscales of constructs rather than composite measures. Thorough reporting also is important when different measures are used to index one construct. The exclusion of findings relating to some (or only part of) measures creates an obligation to provide a rationale beyond a lack of statistical findings for removing them. Avoid the appearance of fishing expeditions.

\section{Use Appropriate Statistical Analyses}

Strong empirical manuscripts use statistical methods that best describe and convey the properties of their data. This means that the Journal embraces flexibility and does not require particular analytical methods. We require analyses that successful researchers in the particular area of study view as reasonable. Sometimes data analyses that do not focus on hypothesis testing could be appropriate. But, whenever possible, report the results of null hypothesis testing. We recognize controversies regarding hypothesis testing and ways of reporting significance levels. But we also recognize that the vast majority of readers best understands research that uses traditional methods of reporting significance levels; and the same is true for highly respected journals that still report traditional significance levels. In this regard, readers benefit from having authors report full significance levels, from .05 to .001 , rather than, as some fields of study require, only .05 . Similarly, trends that are not statistically significant are not significant; they have no place in demonstrating statistical significance. Lastly, even if results are statistically significant, compelling research analyses still need to explore the actual significance of the findings.

\section{Consider Internal Replication}

The social sciences, following the natural sciences, have become very much concerned with replication, especially the apparent lack of it. As a result, some studies report external replication efforts, which are literal replications of the primary features of an original study (e.g., design, methods, and outcomes) in another context, such as another culture, region, or group. Given the challenges inherent in such replications, it has become increasingly common, and expected, that authors consider internal replication, which involves using the same dataset. For such replications, for example, internal replication can be accomplished by dividing the total sample at random into a derivation sample and a cross validation sample and then conducting identical analyses on each. Even with small samples, efforts could be made to determine the extent to which the findings represent a trend in the data.

Despite justified concerns about replication, it is important to maintain perspective. Some data may pose challenges for addressing internal replication. Also, a replication with results that differ from the original study need not indicate that the original one was problematic, as the replication study itself warrants replication. Again, the goal is to help readers understand the level of care put in efforts to construct and interpret reasonable analyses. Also in this regard, effective use of theory and hypotheses grounded in existing research can go a long way toward creating a compelling narrative about the findings and their robustness.

\section{Explain Analyses Transparently}

Poor reporting is inherently problematic as it renders readers unable to follow the manuscript's narrative. Effective manuscripts reveal the statistical methods that 
were used and their appropriateness. If the methods are not widely known, readers benefit from references supporting the impression that the approach is needed and from discussions providing sufficient information to follow (or follow-up on) the analyses. Readers also expect to have relevant analyses conducted and reported if their results are discussed, just as readers expect to have conclusions supported by the data.

Effective reporting gives readers a sense of transparency and credibility. For example, depending on the covariates chosen for a particular model, the results can change dramatically or not change much at all. Yet, decisions about which covariates to include are subjective, which raises the need for transparency about covariate decisions. It is advisable to include the simplest model, the one without controlling for key covariates, either in the main manuscript itself, an appendix, or as a supplement. And, as noted earlier, pre-specifying models in pre-analysis plans helps reveal the relevance of covariates in explaining variation in outcome data. Analyses are effective if they are comprehensive, if readers understand the rationales for them, and if readers can understand what they mean.

Effective manuscripts also recognize that explaining analyses means much more than reporting understandable results, it means reporting them in a way that readers can understand their significance. For example, readers have different strengths when they interpret data; providing simple, uncluttered graphs can effectively help readers visualize complex findings and follow the manuscript's narrative. In addition, non-effects are important to consider. In fact, the Journal has published manuscripts that did not find statistically significant findings when such findings would have been expected. Strong evidence of null effects can be as useful as significant effects. Again, the goal is to offer compelling narratives indicating that the analyses and findings are making original and substantive contributions to the area of study.

\section{Recognize the New Normal}

Journals often place authors in a bind, and this journal provides no exception. Leading journals place increased demands on authors. Yet, journals seem to not make appropriate adjustments for them. For example, some journals require external replication of findings, others require the presentation of multiple models, others require thorough discussions and well-developed theses, while others require reporting on potential conflicts of interest and authors' relative contributions. Our journal makes most of these demands, and it adds to them the need to appeal to a broad audience, which often means elaborating on theories, concepts, and findings. All of that takes space. Yet, journals routinely place tight limits on manuscripts' number of words.

The result of these new demands is that authors, and journals themselves, must make strategic decisions. These decisions require balancing many factors; in fact, they require balancing how to address each of the points made above against each other as well as against many other ones. Journals have sought to address space issues by offering links to supplementary materials. Although these offers may be useful, it is not clear how they address key concerns. Materials not in main manuscripts are not necessarily readily accessible and it is not clear whether readers are interested in reading materials that even the authors viewed as tangential. Similarly, an age of wanting quick answers and of experiencing information overload makes it unwise to expect readers to read exceedingly long manuscripts. In addition, compelling narratives are challenging to maintain when burdened with many side arguments. It is difficult to underestimate the number of critical decisions that make manuscripts strong, and how those decisions involve balancing important factors needed to build solid scientific contributions.

Recognizing these challenges, the Journal has sought to be responsive in the following manner: once manuscripts have survived external review, we no longer have page limits for revisions. Offering more space in the main manuscript clearly is not a cure-all. But, it does mean that the responsibility to make reasonable decisions about what to address still remains where it should be, with the authors. As much as editors seek to guide authors, and as much as authors seek to be responsive to journals' requests, journals need to be supportive as much as possible as authors face the new normal-increased demands from journals, increased choices as to how to address those demands, increased pressure to publish, and increased competition in journal outlets-as they develop and disseminate their findings.

As manuscripts develop, they may well end up more appropriate for unintended journals. This leads to perhaps one of the most important aspects of the new normal: the need to recognize the benefits of rejection. At least for our journal, the vast majority of rejected articles are those that fail to fit squarely enough in the journal's mission. Some rejections come along with multiple, in-depth reviews while other rejections (now well over half of our submissions) are quickly dispatched with comments that highlight why the manuscript would not survive full review. These rejections are important sources of information that can be used positively, as they can become part of the many factors that authors balance to make effective contributions to developmental science. 


\section{Conclusion}

Considering the above points hopefully will help authors produce more competitive manuscripts, either for this journal or another. Authors also are encouraged to consider other editorials relevant to publishing in the Journal (Levesque 2006, 2007, 2011, 2012a, b). And, authors should feel free to consult with the editor before submitting their manuscripts. The Journal's review process quickly moves manuscripts along. But, we recognize that sometimes authors need support before submitting and when revising to help ensure the most favorable editorial outcomes and more effectively develop contributions to the study of adolescence.

Conflict of interest The author reports none.

\section{References}

Levesque, R. J. (2006). Reporting empirical research on adolescence: Reflections on the appeal of coherence, purpose and responsibility. Journal of Youth and Adolescence, 35(1), 1-9.
Levesque, R. J. (2007). Book reviews and the need to take books more seriously. Journal of Youth and Adolescence, 36(8), $1086-1088$.

Levesque, R. J. (2011). The journal of youth and adolescence at 40. Journal of Youth and Adolescence, 40(1), 1-2.

Levesque, R. J. (2012a). On proposing special issues for the journal of youth and adolescence. Journal of Youth and Adolescence, 41(12), 1688-1690.

Levesque, R. J. (2012b). Authorships, collaborations, and acknowledgments: On the increasing responsibility to report relative contributions. Journal of Youth and Adolescence, 41(12), $1685-1687$.

Roger J. R. Levesque Professor of Criminal Justice and (Affiliate) Law at Indiana University, serves as Editor of the Journal of Youth and Adolescence and Adolescent Research Review. 